\title{
Klassifizierung von Modellierungsfällen in modellgetriebenen Decision Support Systemen
}

\author{
Christian Schultewolter
}

\begin{abstract}
Fachgebiet BWL, Management Support und Wirtschaftsinformatik Institut für Informationsmanagement und Unternebmensfübrung, Universität Osnabrück
\end{abstract}

\section{Einleitung}

Decision Support Systeme (DSS) sind nach Alter (1996, S. 225) ursprünglich computer-basierte Systeme, die den Benutzer bei der Identifikation und Auswahl geeigneter Entscheidungsalternativen in Situationen, zu denen keine Erfahrungen oder praktischen Lösungsansätze vorliegen, unterstützen. Dieser Beitrag folgt der etablierten Auffassung der Special Interest Group on Decision Support, Knowledge and Data Management Systems der Association for Information Systems, die DSS in kommunikations-, daten-, dokumenten-, wissens- und modellgetriebene Systeme differenziert (Turban et al. 2007, S. 119). In diesem Kontext werden modellgetriebene Decision Support Systeme (MDSS) synonym auch Spreadsheet-basierte DSS genannt, da Spreadsheet Pakete, wie z. B. Microsoft Excel, oft für die Implementierung solcher Systeme herangezogen werden (Turban et al. 2007, S. 120; Whinston und Holsapple 1996, S. 184; Power und Sharda 2007, S. 1046). Die Hauptaufgabe modellgetriebener DSS besteht in der dynamischen, mehrfachen Analyse oft vektorieller Formeln, die zwischen mehreren Variablen in einem multidimensionalen Modell bestehen (Power und Sharda 2007, S. 1044). Im Vergleich hierzu richten datengetriebene Systeme ihr Hauptaugenmerk auf die Konsolidierung und zeitspezifische Organisation großer Datenmengen, z. B. mittels Data Warehouses (March und Hevner 2007, S. 1031). Leistungsvermögen und Potenzial modellgetriebener DSS werden von der Mächtigkeit des Funktionsspektrums definiert, welches dem Anwender bei der Modellierung zur Verfügung steht. Demnach also den Algorithmen und mathematischen Formeln (Power und Sharda 2007, S. 1045; Sprague und Watson 1996, S. 112), die für die Darstellung realer Situationen zur Verfügung stehen. Der Unterschied beider Systemtypen wird deutlich, wenn man die Zugriffsmöglichkeiten von Formeln und Beziehungen näher betrachtet, die dem Anwender während der Nutzung des Systems zur Verfügung stehen. In datengetriebenen Systemen kann deren Manipulation lediglich im Vorfeld der Erstellung ein- 
zelner Modell-Instanzen (z. B. OLAP-Würfel), z. B. durch Änderung der ETLProzesse, stattfinden. So ist es zwar möglich mehrere Szenarien innerhalb eines OLAP-Würfels abzulegen, jedoch ist es im Nachhinein nicht möglich z. B. die vordefinierten Dimensionen um Weitere zu ergänzen (March und Hevner 2007, S. 1038). Modellgetriebene DSS stellen dem Anwender die gesamte Modelllogik zur Verfügung und ermöglichen dadurch unterschiedliche Analysen mit beliebigen Modellparametern, ohne die Einschränkung vordefinierter Fixwerte. Diese Unterschiede führen zu einem typischen Funktionsspektrum je DSS-Typ. Für MDSS sind hier primär Zielwertsuchen (Goal-seeking), What-if und Sensitivitätsanalysen zu erwähnen.

Panko und Sprague sowie Powell et al. haben in empirischen Studien festgestellt, dass Spreadsheet-Modelle oft Fehler enthalten (1998, S. 337-353; Powell et al. 2008, S. 128-138). Das Auftreten von Fehlern ist hierbei häufig auf eine logischfalsche Modellierung zurückzuführen. Diese hat ihren Ursprung nicht ausschließlich in der Unkenntnis der speziellen Problemsituation und ihrer Entstehung, sondern wird durch eine einschränkende, oft wenig intuitive Modellierungskomponente forciert.

Analog zur Datenmodellierung könnte eine konzeptuelle Modellierungsebene für MDSS, welche eine Modellierungssprache anbietet, Unabhängigkeit von Werkzeug-spezifischen Konzepten und Einschränkungen bieten sowie eine möglichst natürliche Modellierung ermöglichen. Ein positiver Nebeneffekt zeigt sich hier in der Berücksichtigung der klassischen DSS-Forderung die Distanz zwischen Entwickler und Entscheidungsträger zu minimieren (Sprague und Watson 1996, S. 45; Turban et al. 2007, S. 11).

Das übergeordnete Ziel des Forschungsvorhabens, zu dem dieser Artikel einen Beitrag leisten soll, ist die Konstruktion einer solchen konzeptuellen Modellierungsebene für modellgetriebene (Spreadsheet-basierte) DSS. Dieser Beitrag unterbreitet einen ersten Vorschlag von Klassifizierungskriterien für die systematische Beschreibung formelmäßiger Verknüpfungen innerhalb eines Modells.

Wand und Weber (2002, S. 364) beschreiben in ihrer Arbeit ein Framework für die weitere Erforschung von konzeptuellen Modellierungsansätzen. Es besteht aus einer Grammatik, einer Methode, einem Skript und dem Kontext, jeweils bezogen auf konzeptuelle Modellierung. Die Grammatik liefert Konstrukte und Regeln, welche die Vorschriften bei der Kombination von Konstrukten angeben, um reale Zusammenhänge zu modellieren (Wand und Weber 2002, S. 364). Die Methode beschreibt wie reale Zusammenhänge mithilfe der Grammatik modelliert werden können, das Skript stellt ein finales Dokument dar, welches die modellierten Zusammenhänge in Form der Sprache, erzeugt durch die Grammatik, repräsentiert. Der Kontext beschreibt die Begebenheiten und Situationen, in denen konzeptuelle Modellierung herangezogen wird. In diesem Zusammenhang würden die angesprochenen Klassifizierungskriterien einen ersten Schritt zur Erarbeitung einer Grammatik darstellen. 
Eine konzeptuelle Modellierungsebene für MDSS sollte des Weiteren eine nichtprozedurale Modellspezifikation (Mayer 1998, S. 325) unterstützen, da diese oft als Charakteristikum eines DSS beschrieben wird. Konsistente Kriterien für die Klassifizierung verschiedener Beziehungstypen können die Transformation nichtprozedural modellierter Zusammenhänge in korrekte, prozedurale Rechenschritte erleichtern.

Im nächsten Kapitel wird ein Vorschlag von Kriterien zur Klassifizierung unterschiedlicher Beziehungen innerhalb von MDSS ausgearbeitet. Kapitel drei erläutert für jede Beziehung ein vereinfachtes Beispiel und eine Skizze unterstützt dies visuell. Kapitel vier gibt abschließend einen Überblick über die Bedeutung dieses Beitrags im Gesamtkontext des Forschungsvorhabens und präsentiert einen kurzen Überblick über weitere Schritte.

\section{Klassifizierungskriterien von Modellbeziehungen}

\subsection{Grundlagen}

Zentrales Element der vorgeschlagenen generischen Spezifikation ist die (formelmäßige) Beziehung zwischen Modellzellen innerhalb eines diskreten, multidimensionalen Raums. Jede Dimension eines Raums soll generell die gleichen Typen arithmetischer Verknüpfungen anbieten können. Demzufolge sollen Dimensionen, die eine spezielle Aufgabe wahrnehmen oder spezielle Funktionen anbieten, vermieden werden. Dadurch ist es möglich auch große Modelle schnell und leicht verstehen und mit angemessenem Aufwand pflegen zu können. Letztlich kommt diese Forderung der flexiblen Anwendungen von DSS-Funktionen zu gute. So muss beispielsweise sichergestellt werden, dass für eine konsolidierende Ausprägung Jahressumme Werte anderer Dimensionen summiert (z. B. Mengen oder Umsätze) oder als Durchschnitt (z. B. Quoten oder Preise) berechnet werden. Eine ähnliche Forderung findet sich bereits in den zwölf Regeln für OLAP-Systeme von Edgar F. Codd (Regel 6 - Generische Dimensionalität) (Codd et al. 1993, S. 12). Diesem Ansatz folgend ist der Ausdruck Dimension im Weiteren als generischer Begriff zu verstehen. Das bedeutet, dass Kennzahlen und Dimensionen, im Gegensatz zu anderen mehrdimensionalen Modellierungsansätzen verwandter Themengebiete, keine streng getrennten Bestandteile eines Modells mit unterschiedlichen Bedeutungen darstellen (wie z. B. im SDWM (Böhnlein und Ende 2001, S. 32)), sondern Kennzahlen auch qualifizierende Dimension repräsentieren können. Abschließend ist zu erwähnen, dass eine Verknüpfung, die eine bestimmte Logik ausdrückt, lediglich einmal definiert und der Modellierungsprozess nichtprozedural sein sollte, um eine konsistente und nicht redundante Modellierung gewährleisten zu können. 


\subsection{Kriterien und deren Wirkung}

Die Konstruktion einer generischen Spezifikation möglicher Modellbeziehungen bildet die Basis für eine konzeptuelle Modellierungsebene. Dem Framework für die weitere Erforschung von konzeptuellen Modellierungsansätzen von Wand und Weber (2002, S. 364) folgend, müssen Kriterien für eine generische Spezifikation von Modellbeziehungen in MDSS die Modellierung sämtlicher realer Situationen und Zusammenhänge ermöglichen. Die Arbeit von Green (1997, S. 7) erarbeitet für dieses Framework die beiden Kriterien minimale ontologische Überschneidung (MOO - minimal ontological overlap) und maximale ontologische Vollständigkeit (MOC - maximum ontological completeness), welche durch adäquaten Einsatz der Grammatik erreicht werden sollen. MOO drückt aus, dass ein Konstrukt nicht durch andere grammatikalische Konstrukte oder deren Kombination formuliert werden kann. Hierdurch verringert sich die Wahrscheinlichkeit, dass konfliktäre ontologische Repräsentationen eines realen Zusammenhangs auftreten können. Bezogen auf dieses Paper bedeutet dies, dass es für die Modellierung realer Situationen lediglich eine korrekte Möglichkeit (Kriterien-Kombination) gibt. Maximale ontologische Vollständigkeit wird indes erreicht, wenn durch die Kombination der vorhandenen grammatikalischen Konstrukte sämtliche reale Phänomene mittels der Grammatik repräsentiert werden können. Gleichzeitig erhöht sich die Wahrscheinlichkeit, dass eine Domäne komplett dargestellt ist.

Im vorliegenden Beitrag wird jede mögliche zu modellierende Situation durch ein betriebswirtschaftliches Beispiel veranschaulicht. Diese fokussieren vor allem die Darstellung der dimensionalen Differenzierung von Modellbeziehungen sowie die Herausforderung der Transformation von nicht-prozedural modellierten $\mathrm{Zu}$ sammenhängen in automatisiert ausführbare Berechnungsschritte (Rieger 1993, S. 135). Aufgrund dieser Zusammenhänge und der Möglichkeit sämtliche reale Zusammenhänge modellieren zu können, müssen die Klassifizierungskriterien in der Lage sein die charakteristischen, multidimensionalen Strukturen und sich daraus ergebende Konsequenzen für die Transformation zu unterscheiden. Multidimensionale Räume in Kombination mit vektoriellen Formeln führen innerhalb Spreadsheet-basierter Systeme in der Regel zu Überschneidungen von Formeln für gewisse Zellbereiche, die bestimmte Formelreihenfolgen bedingen, und zu speziellen Berechnungsreihenfolgen beteiligter Zellen. Diese beiden Reihenfolgen und deren Unterscheidung sind für das weitere Verständnis eminent wichtig, da sie aus unterschiedlichen Zusammenhängen resultieren. Sie haben ihren Ursprung in speziellen Eigenschaften, die von den Klassifizierungskriterien auf angemessene Art und Weise dargestellt werden müssen. Werden diese Forderungen berücksichtigt, erfüllt eine derartige Spezifikation die Kriterien nach Green sowie Wand und Weber automatisch. Folgende binäre (zwei mögliche Ausprägungen) Kriterien, welche in ihrer Grundstruktur auf Rieger (1993, S. 136) zurückgehen, werden vorgeschlagen: Grad, Homogenität und Simultanität von Dimensionen, die an einer Beziehung beteiligt sind (vgl. Abbildung 1). 


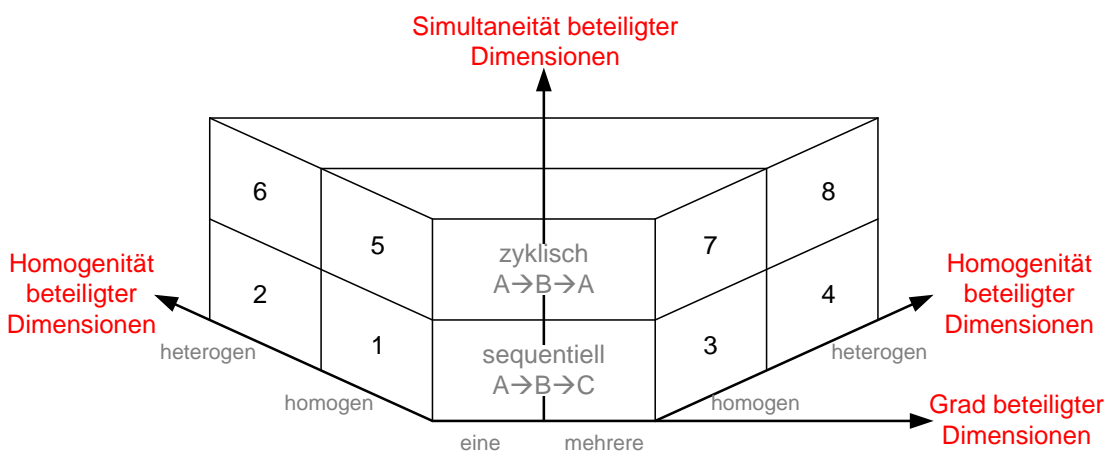

\section{Abbildung 1: Vorgeschlagene Klassifizierungskriterien}

Grad beschreibt die Anzahl von Dimensionen, die an einer Beziehung beteiligt sind. Mögliche Ausprägungen sind hier eine oder mehrere. Hauptaussage dieses Kriteriums ist, ob eine Beziehung intra- oder inter-dimensionaler Natur ist. Das heißt, ob Dimensionen miteinander verknüpft werden oder ob sich sämtliche logische Zusammenhänge auf eine Dimension beschränken. Grad beeinflusst die Vollständigkeit und Konsistenz der Modellspezifikation sowie die Berechnungskomponente beim Herstellen einer korrekten, prozeduralen Berechnungsreihenfolge der Zellen, da in einem multidimensionalen Raum und mehr als einer beteiligten Dimension ein einfaches Sortieren von Dimensionsausprägungen unter Umständen nicht mehr ausreicht. Dimensionsausprägungen können als Variable einer Formel (z. B. Umsatz in einer Formel „Umsatz $=$ Menge $*$ Preis" $)$ verstanden werden. Sowohl die Reihenfolge der Berechnungsformeln für Zellen, die mit mehr als einer Formel belegt sind, als auch die Reihenfolge der Berechnung von Dimensionen im Falle von inter-dimensionalen Formeln (Gradausprägung = mehrere) ist relevant, da Inkonsistenzen zu einem logisch falsch berechnetem Modell führen können. Für die restlichen Zellen, welche lediglich mit einer (oder keiner) intra-dimensionalen Formel belegt sind (z. B. Rohdaten), muss lediglich die intra-dimensionale Berechnungsreihenfolge, die durch einfaches Umsortieren erreicht werden kann, berücksichtigt werden.

Folgendes Beispiel erläutert kurz die Wirkung der Ausprägung Grad: In einem Modell sind die beiden Dimensionen Kennzablen und Zeit enthalten. Auf der Kennzahlen Dimension befinden sich die beiden Formeln „Umsatz $=$ Menge $*$ Preis“ und „Gewinn = Umsatz - Kosten“. Auf der Dimension Zeit ist lediglich eine Summenformel, die die zwei Ausprägungen „2009“ und „2010“ in einer Ausprägung „Summe“ addiert, vertreten. Für die Dimension Kennzahlen muss hier sichergestellt werden, dass der Umsatz vor dem Gewinn berechnet wird, da der Gewinn auf dem Umsatz aufbaut. Bei intra-dimensionalen Formeln ist die Reihenfolge der Dimensionen demnach irrelevant, stattdessen spielt nur die Sortierung der Zellen innerhalb der Dimension eine Rolle. Für inter-dimensionale Referenzen 
muss die Reihenfolge der Dimensionen für alle betroffenen Zellen berücksichtigt werden. Dies ist der Fall, da die Werte referenzierter Zellen, die mit Formeln einer anderen Dimension belegt sind, als der an einer bestimmten Stelle des Berechnungsprozesses aktuellen, bereits vorliegen müssen. Diejenigen Zellen, die nicht durch Kombination mit den Dimensionsausprägungen „2009“ oder „2010“ der Zeit Dimension entstehen, sind zugleich mit der Summenformel aus der Zeit Dimension belegt. Für diese muss ebenfalls die Reihenfolge der Dimensionen, d.h. der Berechnungsformeln aus unterschiedlichen Dimensionen, berücksichtigt werden, um ein korrektes Modell zu erhalten. Generell werden konsolidierende Formeln, wie z. B. die Summenformel der Zeit Dimension, eher am Ende eines Berechnungsprozesses platziert, es gibt jedoch Ausnahmen.

Das Kriterium Homogenität gibt an, ob die logische Struktur eines Modellierungsproblems die Bildung von Intervallen erfordert. Mögliche Ausprägungen sind bomogen, falls keine Fallunterscheidungen nötig sind und beterogen für Fälle, in denen mehrere Formeln unterschieden werden müssen (Intervalle). Dies geht auch aus Abbildung 1: Vorgeschlagene Klassifizierungskriterien hervor. Liegen verschiedene Intervalle für eine Dimensionsausprägung vor, so müssen intra- und interdimensionale Strukturen unterschieden werden. Bei intra-dimensionalen Formeln kann lediglich ein Wert innerhalb derselben Dimension für die Unterscheidung der Formeln verantwortlich zeichnen. Im inter-dimensionalen Fall ist es möglich, dass Ausprägungen weiterer Dimensionen die Anwendung intervallspezifischer Formeln bedingen. Bei mehreren, unterschiedlichen Formeln für eine Dimensionsausprägung kann man sich eine Intervallbildung auch als Partitionierung des Modellraumes vorstellen.

Das dritte und letzte Kriterium Simultanität gibt an, ob simultane Strukturen innerhalb von Formeln oder Referenzen vorliegen. Die möglichen Werte werden hierbei durch syklisch und sequentiell dargestellt. Simultanität besteht, wenn zwei oder mehr Dimensionsausprägungen miteinander zur selben Zeit interagieren, so dass ein Kreisschluss entsteht (Zeit-diskret). In betriebswirtschaftlichen Problemen spielt Simultanität oft eine wichtige Rolle. Die Wahrscheinlichkeit eines Auftretens simultaner Strukturen erhöht sich mit dem Detaillierungsgrad der modellierten Zusammenhänge, da bei mehreren Größen in der Regel auch mehrere Interaktionen innerhalb eines Modells stattfinden (ansonsten würde das Berücksichtigen einer Größe wenig Sinn machen). Simultanität kann sich auch hinter einer Kette formelmäßiger Zusammenhänge verbergen. Auch in solchen Fällen muss jedoch eine gesonderte Rechnung durchgeführt werden, um valide Modelle zu erhalten.

\section{Szenarien}

Der folgende Abschnitt erläutert acht Modellierungsfälle anhand von typischen betriebswirtschaftlichen Beispielen. Die acht Fälle ergeben sich aus der kartesischen Kombination der Ausprägungen der Klassifizierungskriterien untereinander 
(drei Kriterien á zwei Ausprägungen). Die Beispiele sind stark vereinfacht dargestellt und auf zwei Dimensionen limitiert, da dies eine Konzentration auf die wesentlichen, bedeutsamen Zusammenhänge ermöglicht. Diese heheWirkungen werden für jeden Fall mittels eines Ursache-Wirkungs-Diagramms skizziert. Ein Pfeil gibt hierbei die Wirkungsrichtung der Formeln für jeden spezifischen Fall an. Ist dieser diagonal angeordnet, liegt eine inter-dimensionale Beziehung und wenn er vertikal verläuft, liegt eine intra-dimensionale Beziehung vor. Zwei gegenläufige Pfeile stellen wiederum simultane Strukturen dar und eine gestrichelte Linie repräsentiert heterogene Beziehungen, für die eine Fallunterscheidung hinsichtlich der Formeln vorgenommen werden muss. Zweitrangige oder analoge Wirkungen werden innerhalb der Diagramme nicht berücksichtigt.

\subsection{Sequentielle Beziehungen}

Im Folgenden werden zunächst die Fälle 1 bis 4 (vgl. Abbildung 2) behandelt, die sequentielle Strukturen kennzeichnen. Danach werden die Fälle 5 bis 8 (vgl. Abbildung 3), die simultane Strukturen aufweisen, näher erläutert.
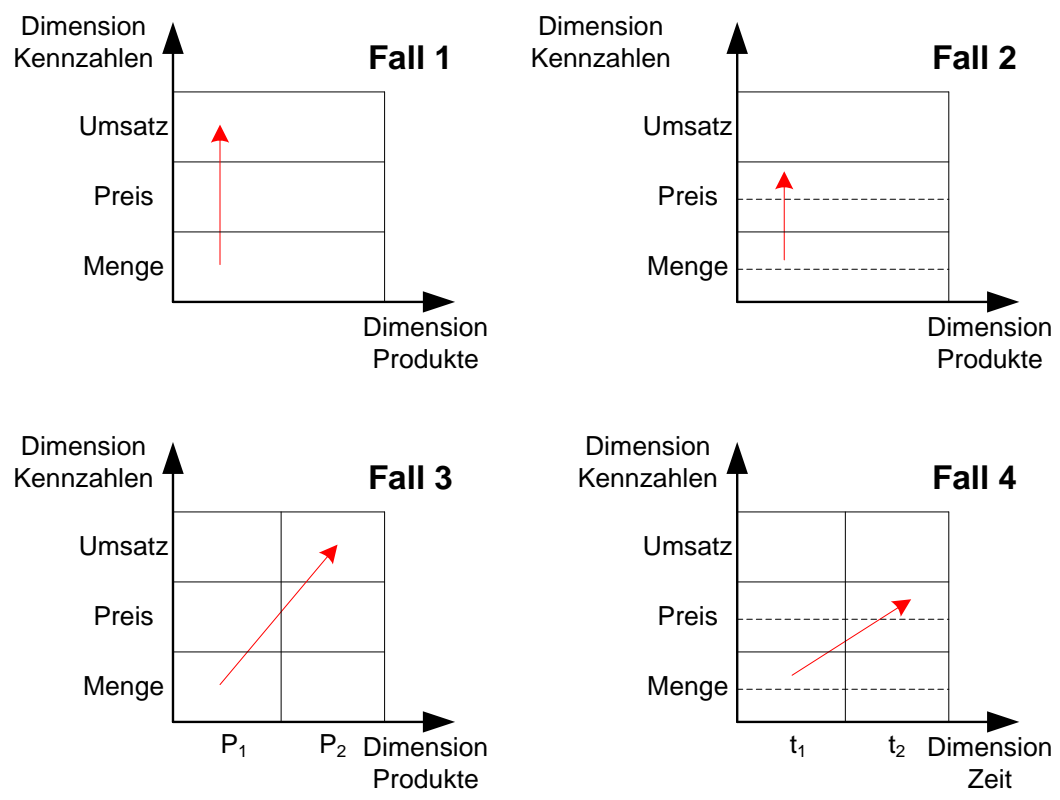

\section{Abbildung 2: Fälle 1 bis 4 mit sequentieller Struktur}

Fall 1: Fall 1 kennzeichnet intra-dimensionale Beziehungen, welche ohne Fallunterscheidung und ausschließlich sequentielle Strukturen aufweisen. Das bedeutet, dass eine intra-dimensionale Formel „Umsatz $=$ Menge $*$ Preis“ auf jede Zelle ange- 
wandt wird, die durch Kombination mit der Dimensionsausprägung „Umsatz“ entsteht.

Die korrekte Reihenfolge der Berechnungsschritte kann durch einfaches Umsortieren der Zellen bzw. ein Sortieren der Formeln für Zellen, die mit mehr als einer Formel belegt sind, erreicht werden. Für alle Zellen, die lediglich mit intradimensionalen Formeln belegt sind, ist die Reihenfolge der Dimensionen irrelevant (vgl. Beispiel für die Wirkung des Klassifizierungskriteriums Grad aus Kapitel 3.2).

Fall 2: Zusätzlich zu Fall 1 werden in diesem Fall Intervalle, das heißt heterogene Formeln eingeführt. Spezifische Werte einer beliebigen Dimensionsausprägung sorgen bei Beziehungen dieser Art dafür, dass unterschiedlich definierte Formeln für die Berechnung ein und derselben Dimensionsausprägung verantwortlich zeichnen. In Bezug auf das Beispiel aus Fall 1 könnte ein Rabattsatz, der ab einer bestimmten Menge gewährt wird, diesen Fall darstellen. Die Formeln könnten wie folgt gestaltet sein: „Umsatz $=$ Menge * Preis * $(1-$ Rabattsatz $) “$. Für eine Menge, die kleiner als 100 ist, würde der Rabattsatz automatisch „0" sein und für Mengen größer 100 durch eine Formel „Menge / 10.000“ berechnet werden.

Für die korrekte Berechnungsreihenfolge muss der Umsatz bei Mengen größer „100“ immer nach dem Rabatt berechnet werden. Für Mengen kleiner „100“ fällt diese Sperre weg, da die Formel für den Umsatz an beiden Stellen des Berechnungsprozesses das gleiche Ergebnis zur Folge hätte.

Fall 3: Während in Fall 1 und 2 ausschließlich intra-dimensionale Referenzen vorliegen beschreibt Fall 3 einen ,echten“ multi-dimensionalen Raum, in dem eine Formel mindestens zwei Dimensionen miteinander in Relation setzt. Eine eingeschränkte Referenz bezüglich bestimmter Werte (Intervalle) liegt jedoch nicht vor.

Bei der Transformation nicht-prozedural modellierter Strukturen in prozedurale Berechnungsschritte muss die Reihenfolge, nach der die Dimensionen abgearbeitet werden, beachtet werden. Wird eine Dimensionsausprägung innerhalb einer Formel, die einer weiteren Dimension entstammt, referenziert, muss die betreffende Zelle zuvor berechnet worden sein. Es wird schnell klar, dass es nicht machbar ist, Zellen einer ganzen Dimension nacheinander zu berechnen, sondern dass eine bestimmte Reihenfolge unabhängig von Dimensionen bei der Berechnung der Zellen hergestellt werden muss.

Ein Beispiel für diesen Typ von Beziehung stellen Formeln für die Abbildung eines mehrstufigen Produktionssystems dar. Es muss sichergestellt werden, dass nicht mehr (Zwischen-)Produkte zur Herstellung eingeplant werden, als es die jeweils vorhergehende Stufe zulässt. So kann zum Beispiel in einer Tischlerei nicht mit 100 Tischen (vgl. Abbildung 2, Fall 3, Größe $\mathrm{P}_{2}$ ) geplant werden, sofern lediglich 100 Tischbeine (vgl. Abbildung 2, Fall 3, Größe $\mathrm{P}_{1}$ ) für den nächsten Produktionslauf vorliegen.

Fall 4 als letzter sequentielle Beziehung ermöglicht zusätzlich zu Fall 3 eine Fallunterscheidung (Intervalle) für bestimmte Werte von Dimensionsausprägungen. Im Vergleich zum Beispiel aus Fall 2 würde die Absatzmenge einer vorange- 
gangenen Periode über die Möglichkeit der Gewährung eines Rabattes entscheiden. Dies hätte zur Folge, dass in der Formel für den Rabattsatz eine Zeitdimension zugegriffen werden müsste.

\subsection{Simultane Beziehungen}

Die folgenden Fälle 5 bis 8 (vgl. Abbildung 3) sind durch simultane Berechnungsstrukturen charakterisiert. Eine simultane Formel muss in einer gesonderten Nebenrechnung, für die es mehrere Strategien gibt, aufgelöst werden. Häufig wird auf Modelle Linearer Programmierung zurückgegriffen, da diese in der Regel auf einfache Art und Weise sehr nah an die optimale Lösung herankommen.
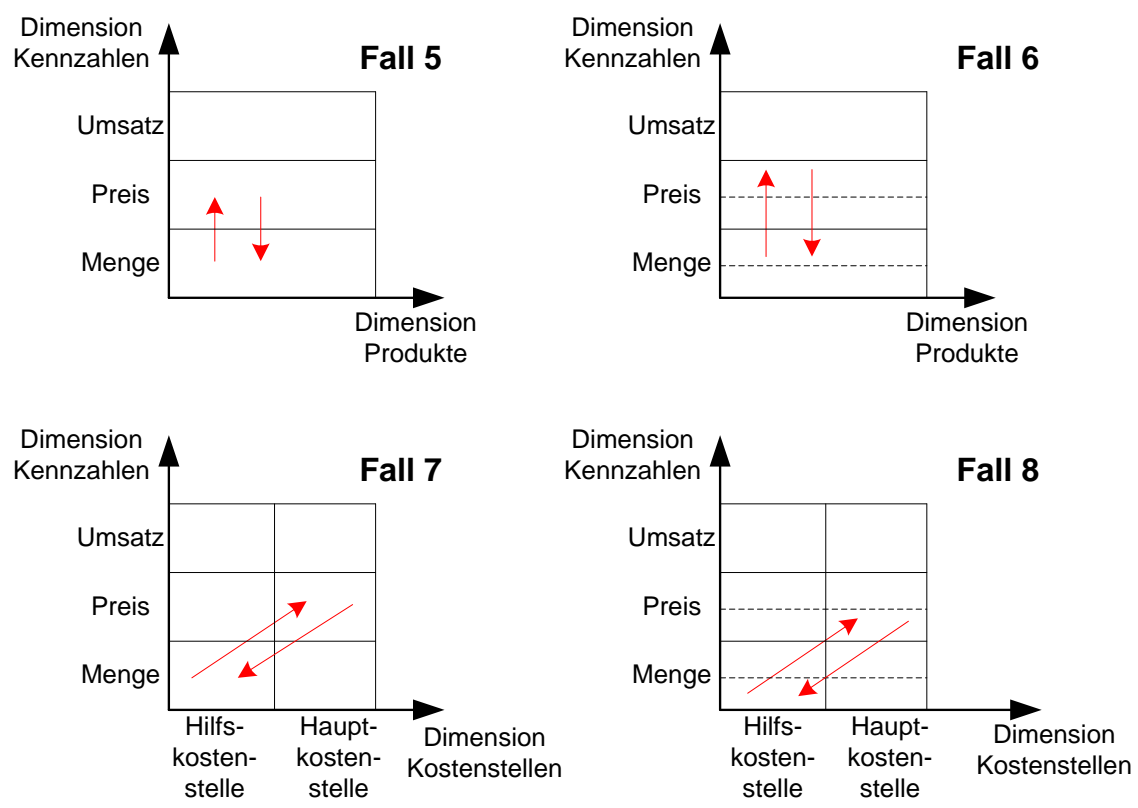

\section{Abbildung 3: Fälle 5 bis 8 mit simultaner Struktur}

Fall 5: Der erste Fall mit simultanen Strukturen berücksichtigt lediglich eine Dimension und keine Fallunterscheidungen für die Formel einer Dimensionsausprägung. Nimmt man das Beispiel aus Fall 1 bezüglich der einfachen Umsatzberechnung wird in diesem Fall nun eine Angebots- und eine Nachfragefunktion abgebildet. Diese beeinflussen sich jeweils gegenseitig, indem die Menge vom Preis (Angebotsfunktion) und der Preis von der Menge (Nachfragefunktion) abhängt.

Beim Etablieren einer korrekten Berechnungsreihenfolge bezüglich Zellen und Formeln muss jede Zelle, die direkt von der simultanen Formel abhängt, in einer Nebenrechnung berechnet werden. In dem Beispiel wären dies die beiden Zellen 
„Preis“ und „Menge“ des abgebildeten Produkts. Die Nebenrechnung müsste nun an einer bestimmten Stelle des gesamten Berechnungsprozesses platziert werden. Diese hängt wiederum von der Sortierung der restlichen Zellen und dem Zusammenspiel dieser mit den simultanen Größen ab. Für Zellen, die mit mehr als einer Formel belegt sind, die aus unterschiedlichen Dimensionen stammen, ist zudem die Formelsortierung (Reihenfolge der Dimensionen) relevant.

Fall 6: Fall 6 führt die Berücksichtigung von unterschiedlichen Formelalternativen für eine Dimensionsausprägung im simultanen Kontext ein.

Zusätzlich zu Fall 5 wären die Angebots- bzw. Nachfragefunktion für ein bestimmtes Mengenintervall (Nachfragefunktion) bzw. ein bestimmtes Preisintervall (Angebotsfunktion) völlig unelastisch. Ab einer bestimmten Menge bzw. einem bestimmten Preis würden sich die Funktionen jedoch auf ähnliche Weise, wie in Fall 5 beschrieben, beeinflussen. Dies hat zur Folge, dass die unterschiedlichen Intervallformeln, sowohl für die Menge als auch für den Preis, an unterschiedlichen Stellen des Berechnungsprozesses platziert werden würden, da die simultanen Zusammenhänge wiederum in einer Nebenrechnung gelöst werden müssen.

Fall 7: Dieser simultane Zusammenhang verbindet Ausprägungen unterschiedlicher Dimensionen miteinander, unterscheidet hierbei jedoch keine Formelalternativen (Intervalle). Die innerbetriebliche Leistungsverrechnung ist ein klassisches Beispiel hierfür. Eine Hilfskostenstelle ist einer Hauptkostenstelle zugeordnet. Auf der einen Seite konsumiert die Hilfskostenstelle Leistungen der Hauptkostenstelle, auf der anderen Seite stellt sie der Hauptkostenstelle Leistung zur Verfügung. Die benötigte Leistung der Hilfskostenstelle beeinflusst den Preis der Hauptkostenstelle und umgekehrt.

Um in diesem Fall eine korrekte Berechnungsreihenfolge der Zellen und Formeln herzustellen, müssen wiederum die Reihenfolge der Dimensionen beachtet und die Nebenrechnung zur Auflösung der simultanen Größen an eine spezifische Stelle des Gesamtprozesses eingegliedert werden.

Fall 8: Der achte und letzte Fall deckt die Möglichkeit von simultanen Formeln in einem multidimensionalen Raum ab, die - im Gegensatz zu Fall 7 - nicht in gleicher Weise für alle Werte der betroffenen Dimensionsausprägung gelten. Erweitert man das Beispiel der innerbetrieblichen Leistungsverrechnung aus Fall 7 dahingehend, dass Kapazitätsgrenzen die Menge an Leistung beschränken, die eine Hilfskostenstelle einer Hauptkostenstelle zur Verfügung stellt, ergibt sich als Konsequenz, dass die Hauptkostenstelle selbige Leistung von außen zukaufen muss. Für einige Werteausprägungen von Elementen der Dimension Kennzahlen gilt also eine unterschiedliche Berechnungsvorschrift (einmal ,intern“ und einmal „extern").

Die unterschiedlichen Intervallformeln müssen an unterschiedlichen Stellen des Berechnungsprozesses platziert werden, da die simultanen Zusammenhänge sich auf ein Intervall beziehen und wiederum in einer Nebenrechnung gelöst werden müssen. Diese muss in den gesamten Berechnungsprozess eingegliedert wer- 
den und zudem ist die Reihenfolge der Dimensionen aufgrund der interdimensionalen Beziehungen relevant.

\section{Ausblick}

Der Beitrag stellt Klassifizierungskriterien einer generischen Spezifikation für modellgetriebene DSS vor, die im Weiteren zu einer konzeptuelle Modellierungssprache weiterentwickelt werden soll, um mit deren Konstrukten und Attributen betriebswirtschaftliche Probleme adäquat beschreiben zu können.

Die mit Hilfe der Modellierungssprache erstellten konzeptuellen Modelle sollen in einem weiteren Schritt als Input eines Übersetzers für Werkzeuge im Bereich modellgetriebener DSS dienen. Das Ziel könnte hier in der Realisierung eines Modellierungsassistenten liegen, der für unterschiedliche Produkte effiziente Modellierungsalternativen vorschlägt.

Bei der Konstruktion der Spezifikation sollen vorrangig die Modellierungsund die Modellberechnungseffizienz berücksichtigt werden. Das Sicherstellen eines minimalen Spezifikationsaufwands hat hier erhöhte Priorität. Bei der Modellberechnung steht der Algorithmus zur Umsetzung der modellierten Formeln im Vordergrund. Die Gewährleistung der Umsetzung nicht-prozedural modellierter Zusammenhänge in prozedurale Berechnungsstrukturen durch komplexe Algorithmen stellt hier eine besondere Herausforderung dar.

Diese Kombination von Modellierung und Berechnung könnte letztendlich zu einer rechnergestützten Modellvalidierungskomponente führen. Denkbar wäre hier die Entwicklung automatischer Prüfungen in Bezug auf Vollständigkeit und Plausibilität von Modellen, die mit unterschiedlichen Produkten umgesetzt wurden (nach deren Transformation). Dem Entwickler könnte bei der Modellierung mit der konzeptuellen Sprache eine Hilfestellung angeboten werden, die z. B. definierte Einschränkungen in der Kombinatorik von Dimensionselementen untersucht.

Als technische Basis zur Umsetzung der Modellierungssprache ist eine Lösung mittels XML angedacht. Eine semantische Attributierung oder die Bildung spezieller, einheitlicher Objekte wurde bereits in anderen Themengebieten, wie z. B. XBRL (eXtensible Business Reporting Language) erfolgreich umgesetzt. Der flexible Charakter von XML bietet viele Möglichkeiten zur Erweiterung und eine geeignete Basis zur Nutzung der Sprache für unterschiedliche Modellierungswerkzeuge.

\section{Literatur}

Alter S (1996) Information Systems: A management perspective. Benjamin / Cummings, Menlo Park.

Böhnlein A, Ende UV (2001) Ein konzeptuelles Data Warehouse-Modell für die Erstellung multidimensionaler Datenstrukturen. In: Gesellschaft für 
Informatik, Fachgruppe 5.10 Informationssystem-Architekturen (Hrsg.), Rundbrief 8. Jahrgang: 25-57.

Codd E, Codd S, Salley C (1993) Providing OLAP to User-Analysts: An IT Mandate. http://www.minet.uni-jena.de/dbis/lehre/ss2005/sem_dwh/ lit/Cod93.pdf. Abruf am 2009-06-15.

Green P (1997) Use of Information Systems Analysis and Design (ISAD) Grammars in Combination in Upper CASE Tools - An Ontological Evaluation. In: Siau K, Wand Y, Parsons J. (Hrsg.), Proceedings of the 2nd CAiSE/IFIP8.1 International Workshop on the Evaluation of Modeling Methods in Systems Analysis and Design (EMMSAD'97), Barcelona: 1-12.

Holsapple C, Whinston A (1996) Decision Support Systems: a knowledge-based approach. West, St. Paul.

March ST, Hevner AR (2007) Integrated decision support systems: A data warehousing perspective. Decision Support Systems 43(3): 1031-1043.

Mayer MK (1998) Future trends in model management systems: parallel and distributed extensions. Decision Support Systems 22(4) 1998: 325-335.

Panko R, Sprague R Jr (1998) Hitting the wall: errors in developing and code inspecting a 'simple' spreadsheet model. Decision Support Systems 22(4): 337353.

Powell S, Baker K, Lawson B (2008) A critical review of the literature on spreadsheet errors. Decision Support Systems 46(1): 128-138.

Power D, Sharda R (2007) Model-driven decision support systems: Concepts and research directions. Decision Support Systems 43(3): 1044-1061.

Rieger B (1993) Der rechnergestützte Arbeitsplatz für Führungskräfte, Habilitationsschrift. Technische Universität Berlin.

Sprague R Jr, Watson H (1996) Decision Support for Management. Prentice-Hall, New-Jersey.

Turban E, Aronson J, Ting-Peng L, Sharda R (2007) Decision Support and Business Intelligence Systems. Pearson, New-Jersey.

Wand Y, Weber R (2002) Research Commentary: Information Systems and Conceptual Modeling - A Research Agenda. Information Systems Research 13(4): 363-376. 